\section{Leptin on the brain}

\section{By Lauren Martz, Staff Writer}

Although the correlation between susceptibility to infection and CNS dysfunction is well documented, ${ }^{1,2}$ the mechanistic link is poorly understood. Now, an international team of researchers may have found the missing puzzle piece-leptin. ${ }^{3}$

Leptin is secreted by adipose cells and acts through the CNS to modulate a variety of biological functions. The hormone's role in food intake and body weight has received the most attention, but some previous work suggested it might also be involved in the body's innate response to infection.

For example, in vitro studies have shown that the hormone activates monocytes by producing inflammatory cytokines, ${ }^{4}$ and it also regulates $\mathrm{T}$ cell activity. ${ }^{5}$

According to Matthias Tschöp, professor of medicine at the Metabolic Diseases Institute of the University of Cincinnati, the classical view has been that leptin might affect immune defenses by acting directly on peripheral immune cells.

However, in a paper published in The Journal of Neuroscience, Tschöp and colleagues now report that leptin acts directly on the CNS to exert systemic control over the immune response. The team also suggests that increasing leptin signaling in brain tissue could help treat sepsis.

The researchers zeroed in on sepsis based on the case histories of patients with congenital leptin deficiency. ${ }^{6}$ Of 12 patients with the extremely rare disorder, 4 died of sepsis. Also, 5 of 16 patients with a loss-of-function mutation in the leptin receptor died of sepsis.

"We chose sepsis since it is a complication and disease state which is not necessarily specific for a certain germ or infection," so thus it was a good backdrop to study how leptin broadly affects the immune response to a general infection, said Tschöp.

In a mouse model of sepsis, the team showed that all leptin-deficient animals died within 72 hours, whereas $30 \%$ of wild-type mice survived for 10 or more days. In the leptin-deficient mice, bacterial colony counts were two orders of magnitude higher following the induction of sepsis than they were in wild-type mice.

In both wild-type and leptin-deficient mice, intraperitoneal administration of leptin reduced kidney and liver damage caused by sepsis compared with administration of saline. In leptin-deficient mice, exogenous leptin significantly decreased bacterial colony counts compared with saline.

The next step was determining whether the effect of leptin was due to activity on the CNS. In both wild-type and leptin-deficient mice, delivery of leptin straight into the CNS increased survival rates and reduced organ damage compared with delivery of saline.

Finally, in leptin receptor-deficient mice, restoration of the receptors only in brain tissue led to survival rates following sepsis challenge that were comparable to those of wild-type mice.

The authors suggested that leptin might help the body fight infection by controlling neutrophil function. Leptin has been shown to induce neutrophil locomotion, and the team saw suppressed phagocytosis in neutrophils in leptin-deficient mice.

Tschöp co-led the team with Charles Caldwell, research assistant professor at the University of Cincinnati. The paper also included researchers from the Ludwig Maximilian University of Munich, Monash University, the Albert Einstein College of Medicine of Yeshiva University, the Cambridge Institute for Medical Research and the German Institute of Human Nutrition.

"The novelty of this work is that leptin accomplishes this through the central nervous system and not direct action on immune cells," said Giuseppe Matarese, senior researcher at the Italian National Research Council's Institute of Experimental Endocrinology and

Oncology.

"This work may be the first to actually show functionally relevant control over systemic immune defense in the CNS in general," said Tschöp.

He said leptin has the potential to treat other infectious conditions, although "which ones specifically leptin would be most helpful against remains to be determined."

"Leptin potentially could be a powerful therapy for any disease state where the patient is immune suppressed," added Caldwell.

\section{Finding new purpose}

Tschöp told SciBX that the next step could be to take leptin analogs and repurpose them for sepsis.

"The good news is that because the drugs are already in clinical testing for other indications, toxicology studies have already been done, highlighting a potential shortcut," he said. "One difficulty going forward is that sepsis is not easy to study in humans. One would need to partner with a pharmaceutical company to help carry out these studies. There are a few companies studying leptin-like molecules for metabolic indications, and it is thinkable that someone with these drugs would consider expanding their program to sepsis as an additional indication."

The company with the most advanced leptin candidate is Amylin Pharmaceuticals Inc. Amylin's pramlintide/metreleptin is in Phase II testing to treat obesity. The compound is a combination of pramlintide, which is an amylin analog, and the leptin analog metreleptin. The company declined to comment.

Late last year, AstraZeneca plc acquired a preclinical leptin modulator program from Swedish Orphan Biovitrum. 
Eli Lilly and Co.'s Xigris drotrecogin alfa recombinant human activated protein C (APC) is the only approved drug to treat sepsis in the U.S., but bleeding risks and a narrow label have limited its use to severely septic patients at high risk of death.

"Sepsis is a condition for which there is no effective treatment. Because there is urgent need for the deadly disease, this novel application for leptin may be given sufficient attention," said Tschöp.

Matarese agreed that moving into human trials is the logical next step for leptin-based therapeutics.

"The work in metabolic indications has effectively shown us that people, unless leptin deficient, do respond very well to leptin, which gives support to use the treatment in other indications," he said. "We propose now giving this compound to patients to fight infection."

Matarese did note that one potential side effect is that leptin or a leptin analog might produce weight loss and anorexia, "which could be particularly dangerous because sepsis patients are already quite ill."

Delivery could be another obstacle.

"There are no methodologies to easily deliver leptin only to the CNS," said Oreste Gualillo, head of the Neuroendocrine Interactions in Rheumatology and Inflammatory Diseases group at the Santiago de Compostela University Clinical Hospital. "It will be important to determine the molecular mechanisms by which CNS leptin mediates the host immune response to sepsis so we can mediate these pathways to improve sepsis outcomes" potentially by other means.
Tschöp said that he and his colleagues are already in talks with undisclosed companies to discuss potential clinical trials with leptinbased therapies. He said his team has not patented the findings.

Martz, L. SciBX 3(19); doi:10.1038/scibx.2010.577

Published online May 13, 2010

\section{REFERENCES}

1. Dziedzic, T. et al. Crit. Care 8, 266-270 (2004)

2. Meisel, C. et al. Nat. Rev. Neurosci. 6, 775-786 (2005)

3. Tschöp, J. et al. J. Neurosci.; published online April 28, 2010; doi:10.1523/JNEUROSCI.4875-09.2010

Contact: Matthias H. Tschöp, University of Cincinnati, Cincinnati, Ohio e-mail: matthias.tschoep@uc.edu

4. Santos-Alvarez, J. et al. Cell. Immunol. 194, 6-11 (1999)

5. Lord, G. et al. Nature 394, 897-901 (1998)

6. Ozata, M. et al. J. Clin. Endocrinol. Metab. 84, 3686-3695 (1999)

\section{COMPANIES AND INSTITUTIONS MENTIONED}

Albert Einstein College of Medicine of Yeshiva University, New York, N.Y.

Amylin Pharmaceuticals Inc. (NASDAQ:AMLN), San Diego, Calif. AstraZeneca plc (LSE:AZN; NYSE:AZN), London, U.K. Cambridge Institute for Medical Research, Cambridge, U.K. Eli Lilly and Co. (NYSE:LLY), Indianapolis, Ind.

German Institute of Human Nutrition, Potsdam-Nuthetal, Germany Ludwig Maximilian University of Munich, Munich, Germany Monash University, Melbourne, Australia

National Research Council, Napoli, Italy Santiago de Compostela University Clinical Hospital, Santiago de Compostela, Spain

Swedish Orphan Biovitrum (SSE:BVT), Stockholm, Sweden University of Cincinnati, Cincinnati, Ohio 